\title{
Produire la santé, produire la sécurité
}

Récupérations et compromis dans le risque des manipulatrices en radiothérapie

Ensuring health, ensuring safety: risk management by radiographers working in radiotherapy

\section{Adélaïde Nascimento et Pierre Falzon}

\section{(2) OpenEdition}

\section{Journals}

Édition électronique

URL : http://journals.openedition.org/activites/2225

DOI : 10.4000/activites. 2225

ISSN : $1765-2723$

Éditeur

ARPACT - Association Recherches et Pratiques sur les ACTivités

Référence électronique

Adélaïde Nascimento et Pierre Falzon, «Produire la santé, produire la sécurité », Activités [En ligne], 6-2 I octobre 2009, mis en ligne le 15 octobre 2009, consulté le 20 avril 2019. URL : http:// journals.openedition.org/activites/2225 ; DOI : 10.4000/activites.2225

\section{(c) (i) (9)}

Activités est mis à disposition selon les termes de la licence Creative Commons Attribution - Pas d'Utilisation Commerciale - Pas de Modification 4.0 International. 


\title{
Produire la santé, produire la sécurité : Récupérations et compromis dans le risque des manipulatrices en radiothérapie
}

\author{
Adélaïde Nascimento \\ Cnam. Centre de Recherche «Travail \& Développement» (CRTD) - Equipe Ergonomie \\ 41, rue Gay-Lussac. 75005 Paris. \\ IRSN. Service d'Etudes des Facteurs Humains. 92262 Fontenay aux Roses Cedex \\ adelaide.nascimento@cnam.fr \\ Pierre Falzon \\ Cnam. Centre de Recherche «Travail \& Développement » (CRTD) - Equipe Ergonomie \\ pierre.falzon@cnam.fr
}

\begin{abstract}
Ensuring health, ensuring safety: risk management by radiographers working in radiotherapy. Radiographers working in the control room are the last line of defence in radiotherapy before the actual treatment. They have two main goals: (i) ensuring patient health by providing daily care and avoiding appointment cancellations and (ii) ensuring patient safety by providing care under the prescribed conditions and avoiding incidents and accidents. Conflicts between these two goals may arise in non-nominal situations. The aim of this paper is to highlight the trade-offs radiographers sometimes make by analysing how they managed 59 nonnominal situations. In 47 of the situations, the radiographers resolved the conflict without being forced to make "sacrifice decisions" in support of one or other of the two goals. In the 12 remaining situations, the conflict management was accomplished by a trade-off between the goals of patient health and patient safety. When making sacrifice decisions, radiographers take into account the expected risks and benefits for the patients on the one hand, and the treatment phase, along with knowledge about co-workers' behaviour and patient satisfaction, on the other.' satisfaction.
\end{abstract}

KEYWORDS

Conflict, trade-off, risk, health, safety, radiographers, patients, radiotherapy

\section{Introduction}

Sept accidents de radiothérapie ont été récemment déclarés à l'Autorité de Sûreté Nucléaire (ASN) française. Les rapports d'inspection mettent en évidence, entre autres, un manque de culture de sécurité (Peiffert, Simon, \& Eschwege, 2007). Ceci a conduit le Ministère de la Santé à annoncer un ensemble de mesures nationales visant à améliorer la sécurité des pratiques, la qualité des soins et la vigilance en radiothérapie. Cette étude répond ainsi à une demande sociale d'amélioration de la culture de sécurité dans ce domaine médical.

Chaque année en France, environ 180000 patients atteints de pathologies cancéreuses bénéficient d'une radiothérapie. Si cette technique contribue notamment à l'amélioration de la prise en charge des patients (40\% des malades guéris ont été traités par radiothérapie), elle porte des risques qui peuvent conduire à des conséquences graves sur la santé des patients comme en témoignent les derniers accidents survenus en France (SFRO, 2008). 
Fort heureusement, les accidents de radiothérapie constituent des événements rares. Une vingtaine d'accidents graves ont été recensés dans le monde depuis 1974 (Cosset \& Gourmelon, 2002). Ces accidents présentent deux caractéristiques importantes en termes d'impact sur la sécurité des patients: ils ne touchent que rarement un seul patient et l'empan temporel entre la survenue et la découverte du dysfonctionnement peut varier de quelques minutes à quelques années. Prenons deux exemples pour illustrer ces caractéristiques.

Au Royaume-Uni, suite à un changement de technologie, un accident entraînant un sous-dosage a eu lieu en 1982 et n'a été découvert qu'en 1991: 1045 patients ont été sous-dosés de 20 à $30 \%$ de la dose prescrite par le radiothérapeute (Cosset \& Gourmelon, ibid.). Plus récemment (2005), dans un accident de radiothérapie survenu à Epinal (France), environ 4500 patients sont susceptibles d'avoir reçu un surdosage de $7 \%$, c'est-à-dire $2 \%$ supérieur à la limite tolérée (Peiffert, Simon, \& Eschwege, 2007).

L'ampleur des conséquences de tels accidents souligne la nécessité de la détection et de la récupération des écarts avant qu'ils n'aient des résultats négatifs sur la sécurité des patients.

Dans le processus de préparation et d'administration des séances de radiothérapie, chaque étape et ses interfaces porte un risque de défaillance qui pourra aboutir à un écart entre la dose prescrite par le radiothérapeute et celle que sera effectivement délivrée au patient (Nascimento \& Falzon, 2008a). En connaissance de ces risques imminents, les professionnels du domaine mènent des démarches d'assurance qualité lors de la mise en service d'un nouveau système ou en contrôlant régulièrement les plans de traitement individuels (Rosenwald, 2002). Les divers acteurs sont censés, en plus de la réalisation des tâches qui sont propres à leurs métiers, vérifier la conformité de l'ensemble des données relatives au traitement.

Les manipulatrices ${ }^{1}$, professionnels responsables de l'administration des traitements, ont un rôle particulier à jouer dans les tâches de contrôle et de récupération des écarts car elles représentent la dernière barrière de défense avant l'administration du traitement et par conséquent avant la possible survenue d'un accident portant atteinte au patient. En effet la « barrière de défense » fait référence au process, à la succession d'étapes dans la chaîne de production. De plus, d'autres accidents de nature différente peuvent intervenir à d'autres moments. Les manipulatrices doivent répondre ainsi à au moins deux objectifs-clés de leur métier: produire la santé des patients, en administrant le traitement, et produire la sécurité des patients, en administrant le traitement correct, dans les conditions de sécurité prescrites. Les compromis trouvés par les manipulatrices pour répondre à ces objectifs s'inscrivent dans un contexte de risques et de contraintes temporelles fortes. Les premiers sont inhérents à ce type d'activité de soins et les deuxièmes sont imposées par la nature de la pathologie (nécessité de traiter au plus tôt possible, et tous les jours) et par la planification «serrée » des séances (toutes les 15 minutes).

Dans cet article, nous nous intéresserons à l'analyse de l'activité des manipulatrices dans la gestion du compromis entre "produire la santé » et "produire la sécurité » des patients, la gestion des aléas pouvant amener à des arbitrages dans le risque entre ces deux objectifs. Cette analyse focalisée sur un métier et un poste particuliers implique néanmoins une présentation générale de la 《chaîne $»^{2}$ de traitement en radiothérapie, le travail de l'ensemble des intervenants ayant un fort impact sur les activités en bout de chaîne.

1. La profession est majoritairement exercée par des femmes $(70 \%)$. Pour cette raison nous utiliserons le terme au féminin.

2. Le terme « chaîne de traitement » reprend la terminologie utilisée par les professionnels de la radiothérapie. 


\section{1.- La chaîne du traitement en radiothérapie}

\section{1.- Des rayons qui peuvent guérir : la technique en radiothérapie}

La radiothérapie constitue, avec la chirurgie et la chimiothérapie, un des trois principaux moyens de traitement des cancers. Cette technique médicale consiste à irradier un volume tumoral à l'aide d'un faisceau de rayonnement ionisant (en général photons ou électrons). Le faisceau d'irradiation, généralement produit par un accélérateur de particules, est directement dirigé vers la tumeur et l'énergie transférée aux cellules cancéreuses finit par les détruire. L'objectif majeur de la thérapie est d'atteindre une plus grande concentration de la dose prescrite dans le volume tumoral tout en limitant l'irradiation des organes sains proches.

En pratique ceci est accompli par la réalisation de séances journalières consistant à délivrer des fractions de la dose totale, à travers diverses techniques qui déterminent la géométrie des faisceaux d'irradiation et les paramètres des appareils de traitement. L'informatique a fait progresser les techniques de traitement, la précision et l'efficacité de la dose. Ainsi, les moyens technologiques en radiothérapie ne cessent d'évoluer, que ce soit au niveau de l'imagerie médicale ou des logiciels de planification et de délivrance de séances (R. Cook, Nemeth, \& Dekker, 2008). À cause des variabilités liées au type et à la taille de la tumeur, à l'âge et aux caractéristiques morphologiques des patients, à la disponibilité des accélérateurs, etc. le traitement en radiothérapie est une construction unique pour chaque patient. Sont également engagés dans ce processus divers professionnels issus de quatre métiers.

\section{2.- L'engagement d'acteurs multiples}

Compte tenu de la complexité des traitements en radiothérapie, la distribution du travail se fait en fonction des compétences de quatre types de professionnels, tous directement engagés dans la chaîne de production des soins (SFRO, 2008):

- Le radiothérapeute, qualifié par l'ordre des médecins, est titulaire d'un Certificat d'Etudes Spécialisées (CES) ou Diplôme d'Etudes Spécialisées (DES) en radiothérapie. Il s'agit du médecin responsable de l'acte qui inclut la prescription de la dose totale par volume tumoral ainsi que les limites de dose acceptables par organes sains à proximité de la tumeur, la technique d'irradiation à utiliser, la validation du plan de traitement, des calculs de dosimétrie, et la surveillance du patient pendant et post-traitement (à travers notamment les consultations hebdomadaires et de fin de traitement);

- Le physicien médical est titulaire d'un Diplôme de qualification en physique médicale et a pour rôle de "garantir que la dose de rayonnements reçue par les tissus faisant objet de l'exposition correspond à celle prescrite par le radiothérapeute demandeur » (ASN 2007). Ceci se fait à travers la planification des traitements, processus qui détermine comment irradier un patient particulier. Le physicien médical peut être assisté par des dosimétristes, ce qui n'est pas toujours le cas;

- Le dosimétriste participe avec le physicien médical à la construction de la dosimétrie clinique et à l'optimisation du plan de traitements. Comme aucun texte réglementaire ne définit ni la formation, ni la qualification ni les actes qui peuvent être effectués par un dosimétriste, tout travail réalisé par ce professionnel doit être vérifié et validé par un physicien médical;

- La manipulatrice d'électroradiologie médicale est titulaire d'un Diplôme d'Etat ou d'un Brevet de Technicien Supérieur (BTS). Elle est la seule habilitée, sous la responsabilité du radiothérapeute, à l'administration des séances d'irradiation. La manipulatrice est ainsi le membre de l'équipe soignante qui reçoit et qui traite le patient quotidiennement et devient de ce fait un interlocuteur privilégié pour celui. Cette professionnelle participe également en amont de la chaîne aux phases d'acquisition de données anatomiques du patient et de simulation de son 
positionnement lors des séances.

\section{3.- Un ensemble d'étapes pour un traitement sur mesure}

La préparation et l'administration des séances de traitement en radiothérapie se font à travers une succession d'étapes de natures différentes, engageant les professionnels présentés ci-dessus. Treize étapes constituent une traduction de la prescription médicale par chaque métier ${ }^{3}$ (tableau 1).

\begin{tabular}{|c|c|c|}
\hline Phases & Étapes & Professionnels \\
\hline \multirow{2}{*}{ Simulation } & $\begin{array}{l}\text { Acquisition des données anatomiques et de positionnement } \\
\text { du patient }\end{array}$ & $\begin{array}{l}\text { Manipulatrices - poste de } \\
\text { simulation }\end{array}$ \\
\hline & Acquisition d'imagerie 3D & $\begin{array}{l}\text { Manipulatrices - poste } \\
\text { d'imagerie }\end{array}$ \\
\hline \multirow{6}{*}{$\begin{array}{l}\text { Préparation } \\
\text { du plan de } \\
\text { traitement }\end{array}$} & Préparation du dossier informatique pour le radiothérapeute & Dosimétriste \\
\hline & Choix du volume à irradier & Radiothérapeute \\
\hline & Conception de la dosimétrie & Dosimétriste \\
\hline & Evaluation et validation de la dosimétrie & Radiothérapeute \\
\hline & Evaluation et validation de la dosimétrie & Physicien médical A \\
\hline & Transfert des données vers le poste de traitement & Physicien médical A \\
\hline \multirow{4}{*}{$\begin{array}{l}\text { Réception } \\
\text { du plan de } \\
\text { traitement et } \\
\text { vérification de } \\
\text { conformité }\end{array}$} & $\begin{array}{l}\text { Vérification de conformité du dossier papier par rapport à } \\
\text { l'informatique + ajout des données sur le positionnement } \\
\text { du patient en poste de traitement + « appropriation du } \\
\text { dossier » }\end{array}$ & $\begin{array}{l}\text { Manipulatrices - poste } \\
\text { d'administration }\end{array}$ \\
\hline & Contrôle indépendant sur la dosimétrie & Physicien médical B \\
\hline & $\begin{array}{l}\text { Image de contrôle de positionnement du patient en poste de } \\
\text { traitement - séance } 0 \text {, sans irradiation }\end{array}$ & $\begin{array}{l}\text { Manipulatrices - poste } \\
\text { d'administration }\end{array}$ \\
\hline & Evaluation et validation de l'image de contrôle & Radiothérapeute \\
\hline $\begin{array}{l}\text { Administration } \\
\text { du traitement }\end{array}$ & Séance 1, irradiation & $\begin{array}{l}\text { Manipulatrices - poste } \\
\text { d'administration }\end{array}$ \\
\hline
\end{tabular}

Tableau 1: Etapes de préparation et d'administration du traitement

Table 1: Phases of treatment preparation and administration

Ce fonctionnement suppose une bonne compréhension/représentation des étapes et leurs contenus par les acteurs successifs. Les étapes d'évaluation et de validation sont nécessaires au déclenchement de l'étape suivante, ce qui présume également une bonne synchronisation opératoire, c'est-à-dire la garantie de la répartition des tâches entre les partenaires de l'activité collective et son séquencement performant (Darses \& Falzon, 1996).

Suite à la prescription médicale, les plans de traitements sont construits à partir d'images tridimensionnelles issues de la phase de simulation et d'acquisition de données anatomiques. Cette phase de préparation du traitement comprend un système graphique informatisé, conçu à partir d'algorithmes complexes, qui permet de générer et d'affiner de manière interactive la proposition de plan qui sera envoyée au radiothérapeute (Cook, Nemeth \& Dekker, 2008).

Le plan de traitement est ensuite transféré vers le poste d'administration de la dose. Les derniers contrôles avant la mise en place des séances sont réalisés par les manipulatrices et par un physicien médical B (cf. tableau 1), différent de celui qui a proposé le plan. Dans la majorité des cas de cancers, à la première séance avec le patient aucune irradiation n'est réalisée. Cette étape consiste à s'assurer que le positionnement prévu au poste de simulation est reproductible en poste d'administration et se termine par la validation du radiothérapeute sur les images de contrôle réalisées à cet effet. Une fois tous les paramètres contrôlés, la séance d'irradiation peut être réalisée sous la responsabilité des

3. L'étape 2 est nécessaire seulement pour la radiothérapie dite conformationnelle. 
manipulatrices.

\section{2.- L'administration des soins par les manipulatrices : produire la santé et la sécurité des patients}

\section{1.- La réalisation des séances}

Les manipulatrices sont les responsables de l'administration des soins en radiothérapie. Sur le poste d'administration, elles suivent les procédures résultant de la prescription médicale.

Auprès des patients, avant chaque première séance, les manipulatrices doivent s'assurer qu'ils ont bien compris le déroulement de la radiothérapie: le calendrier, la durée des séances, la surveillance médicale hebdomadaire, l'indolence de l'irradiation, l'importance d'une immobilisation absolue, les réactions tissulaires éventuelles, la présence d'un interphone et d'une caméra permettant la communication et la surveillance pendant l'irradiation, etc. (Georgin \& Barret, 2006). Le patient constitue également une source d'information importante pour les manipulatrices: l'écoute et l'observation clinique des patients sont utiles à la détection précoce des effets non attendus de l'irradiation.

Une séance de radiothérapie dure environ quinze minutes. Dix minutes sont destinées à la mise en place du patient en salle d'irradiation. Les cinq minutes restantes sont utilisées pour l'activation des faisceaux programmés informatiquement. Cette manipulation se fait entre l'ordinateur et le pupitre de la salle de commande. Dans la majorité des cas, entre chaque faisceau, les manipulatrices reviennent en salle d'irradiation, l'objectif étant le réglage des paramètres (position de la table, par ex.) pour le faisceau suivant.

Les séances se déroulent sous une forte pression temporelle, dictée par la charge de travail mais aussi par l'urgence des traitements en cancérologie. En effet, une fois le traitement décidé, il doit être mis en œuvre le plus vite possible. La liste d'attente des services de radiothérapie constitue une des préoccupations des médecins, car elle peut aboutir à une perte de chance due à la progression de la tumeur entre le diagnostic et le début du traitement (Lopez, Calagaru, Lamproglou, Boskos, Taillibert, Simon, et al., 2008). L'interruption des séances peut également avoir un effet négatif sur la santé des patients.

En moyenne, 40 patients sont traités par jour et par appareil de radiothérapie. Dans le planning des séances, les patients se succèdent les uns après les autres toutes les 15 minutes. Cette durée par séance est standardisée et ne prend pas en compte la complexité des traitements. Bien évidemment, les traitements ne sont pas égaux entre eux et des situations de retards peuvent apparaître. À ce contexte de charge se rajoutent les risques d'erreurs, propres à la technique des rayonnements ionisants: le risque de sous-irradier ou sur-irradier un patient, le risque de se tromper de patient, le risque de délivrer le traitement au mauvais organe, etc.

L'objectif du travail des manipulatrices dans un tel contexte est double et consiste à assurer l'administration des soins journaliers, évitant les annulations de séances (produire la santé des patients) mais aussi assurer que l'administration des soins se fasse dans les conditions prescrites, évitant les incidents/accidents (produire la sécurité des patients). Ces objectifs ne sont pas contradictoires - on peut produire de la santé en sécurité - mais peuvent entrer en conflit en cas d'aléas, ou du fait d'une forte pression temporelle. Il convient donc de les distinguer.

\section{2.- Produire la santé des patients}

Produire la santé des patients, c'est irradier la zone adéquate, et seulement celle-ci, pendant une durée et avec une dose de rayons prescrites. Toute irradiation se définit par trois paramètres dans lesquels le facteur temps est fondamental. Le premier est la dose totale à délivrer, exprimée en Gray (Gy). Le deuxième correspond au fractionnement, c'est-à-dire le nombre de fractions pour une dose 
totale donnée. Le fractionnement classique est de 5 séances de 1,8 à $2 \mathrm{~Gy} / \mathrm{semaine}$, et l'intervalle entre deux fractions joue un rôle important dans la réparation des lésions des cellules saines, celles-ci étant moins radiosensibles que les cellules cancéreuses. Le troisième paramètre d'une irradiation est l'étalement de la dose et correspond au nombre de jours entre la première et la dernière séance de radiothérapie (Chapet \& Mornex, 2006).

Le facteur temps n'étant pas aléatoire, l'enjeu pour les manipulatrices est de réaliser les traitements prescrits à tous les patients du jour, dans un but d'optimiser leurs chances de guérison.

\section{3.- Produire la sécurité des patients}

Les erreurs en radiothérapie se caractérisent par un écart à la prescription médicale: erreur sur le patient, erreur sur le site anatomique à irradier, absence de protection des sites à ne pas irradier, différences par rapport à la dose prescrite, mauvaise distribution de la dose, mauvais fractionnement de la dose. Ces écarts peuvent comprendre des surdosages ou des sous-dosages et ils seront considérés accidentels s'ils dépassent $5 \%$ de la dose prescrite ou s'ils sont inférieurs à $25 \%$ de celle-ci (CIPR, 2000).

En connaissance de ces risques, la période avant la première séance de traitement englobe un ensemble de vérifications de la conformité des différents éléments constitutifs du plan de traitement. Les manipulatrices doivent s'assurer que tous les moyens matériels et informationnels sont disponibles pour le traitement en question et que tous les accords pour le traitement, notamment les validations des plans de traitement, ont été délivrés.

\section{4.- Le compromis dans le risque, entre santé et sécurité des patients}

Comme souligné plus haut, les deux objectifs alloués aux manipulatrices ne sont pas contradictoires. En effet, si toutes les conditions prescrites sont remplies avant le début de l'irradiation ces dernières vont délivrer les soins aux patients en répondant aux objectifs de production de la santé et de la sécurité.

Cependant, un conflit entre ces deux objectifs peut apparaître en situation de non conformité. Par exemple, si les accords nécessaires pour la validation du plan de traitements n'ont pas été délivrés, les manipulatrices se retrouvent face à un conflit entre santé et sécurité des patients. D'un côté, la réalisation de la séance d'irradiation avec un plan de traitement non validé constitue une prise de risque pour la sécurité des patients. D'un autre côté, l'annulation de la séance d'irradiation - car plan de traitement non validé - constitue une prise de risque pour la santé des patients puisque le traitement ne sera pas délivré au jour prescrit.

La notion de conflit de buts a déjà été développée en ergonomie (Caroly \& Weill-Fassina, 2004 ; Toupin, 2008). Dans leur recherche menée auprès des guichetiers de la poste, Caroly et Weill-Fassina (ibid.) montrent que les conflits entre les différentes logiques de la relation de services engendrent des «situations critiques » porteuses de deux risques principaux pour les opérateurs: le mécontentement des clients ou de la hiérarchie. Dans notre cas, ce sont les situations non nominales/ « critiques » qui engendreront un conflit entre les deux buts d'action des manipulatrices: produire la santé ou produire la sécurité des patients.

Gomes, Woods, Carvalho, Huber et Borges (2009) reprennent le concept de « jugement de sacrifice » - développé par Woods (2006) à l'échelle de l'organisation - pour parler du compromis sécurité/ productivité découlant de la gestion des conflits de buts chez des pilotes d'hélicoptère. Si les pilotes rapportent officiellement un problème détecté dans l'appareil, ils répondent à l'objectif de sécurité mais se privent de l'hélicoptère (et des gains pour la compagnie) durant la période de maintenance. Les décisions de sacrifice des pilotes dépendent en réalité de la durée de la maintenance par rapport à la gravité de la panne.

Nous faisons l'hypothèse que l'activité des manipulatrices face aux situations non nominales est fon- 
dée sur des stratégies de gestion de conflit entre les buts de santé et sécurité. D'une part elles font des régulations pour récupérer les écarts commis au poste mais également venus d'ailleurs (collègues, matériels, organisation) afin d'éviter les décisions de sacrifice issues du conflit de buts. D'autre part, en cas d'impossibilité d'éviter le conflit, leurs stratégies sont basées sur un compromis entre risque et bénéfice escompté : est-il préférable qu'un patient n'ait pas son traitement du jour et ainsi éviter le risque de réaliser un traitement erroné? Ou est-il préférable qu'un patient ait son traitement du jour malgré le risque que ce traitement soit erroné?

À travers une recherche empirique, il sera présenté dans ce texte les stratégies mises en jeu par les manipulatrices pour répondre aux objectifs de «produire la santé » et " produire la sécurité » des patients. C'est à partir des analyses de la gestion de situations non nominales que les compromis dans le risque réalisés par les manipulatrices en radiothérapie seront mis en évidence dans cet article.

\section{3.- Contexte de l'étude}

L'étude a été menée dans deux établissements privés participant au service public hospitalier, localisés en région Parisienne. La demande n'émerge pas directement de ces deux établissements, mais étant conscients des besoins en termes d'amélioration de la sécurité des soins, ces sites se sont portés volontaires pour notre étude. Ces établissements sont distincts en termes de taille, de formes de financement, d'accès à des avancées technologiques, de nombre d'appareils et de personnel, de développement de culture sécurité. Le service de radiothérapie du terrain B est un plus petit service comparé au service de radiothérapie du terrain A (cf. tableau 2).

Par jour, chaque poste d'administration du traitement fonctionne pendant 10 heures et reçoit environ 40 patients.

\begin{tabular}{|c|c|c|}
\hline Ressources matérielles et humaines & Terrain A & Terrain B \\
\hline Postes de simulation du traitement & 2 & 1 \\
\hline Postes d'imagerie médicale & 2 & 1 \\
\hline Postes de préparation du plan de traitement & 2 & 1 \\
\hline Postes d'administration du traitement & 5 & 3 \\
\hline Radiothérapeutes & 14 & 6 \\
\hline Physiciens médicaux & 8 & 2 \\
\hline Dosimétristes & 5 & 1 \\
\hline Manipulatrices & 24 & 13 \\
\hline Cadres de santé & 3 & 1 \\
\hline Qualiticiens & 1 & 1 \\
\hline
\end{tabular}

Tableau 2: Ressources matérielles et humaines des services de radiothérapie observés

Table 2: Staff and equipment of radiotherapy services which were observed

\section{4.- Méthodes}

Les méthodes classiques d'analyse de l'activité utilisées en ergonomie ont été mises en œuvre. Elles comprennent des phases d'observations ouvertes et systématiques sur les deux services de radiothérapie citées plus haut ainsi que des entretiens ouverts avec les professionnels du domaine.

\section{1.- Méthodes de recueil de données}

Une première phase de la recherche était destinée à la familiarisation avec le domaine de la radiothé- 
rapie. Des observations ouvertes ont été réalisées pendant 8 semaines sur les divers postes du service de radiothérapie du terrain A: simulateurs ( 2 semaines), scanner (3 jours), salles de dosimétrie (2 semaines), salle de consultation médicale ( 2 jours) et postes d'administration de la dose (3 semaines). Le recueil de données a été réalisé par la technique " papier-crayon ». Cette même démarche a été réalisée dans le terrain $\mathrm{B}$. Au total 180 heures d'observations ouvertes ont été réalisées.

Ces observations ont permis de repérer les problèmes les plus fréquents relatifs aux dysfonctionnements humains et organisationnels de la chaîne de traitement en radiothérapie. Elles ne permettent pas de quantifier les dysfonctionnements ni d'apprécier les conduites tenues par les divers professionnels en temps réel. Des observations systématiques sur les deux terrains ont été conduites à cette fin.

Les observations systématiques ont porté sur l'activité des manipulatrices en poste d'administration des traitements pour deux raisons. D'une part c'est ce poste qui rend le mieux compte du produit final de la construction collective des traitements, à travers les spécifications concrètes de prise en charge des patients. Ainsi, c'est au poste de traitement que l'ensemble des écarts issus en amont ont la plus grande visibilité. D'autre part, on s'est intéressé à l'activité de ces professionnels dans la gestion des écarts commis au poste mais également à ceux venus d'ailleurs (collègues, matériels, organisation) afin de mieux comprendre les régulations mises en place pour gérer le conflit entre produire la santé et produire la sécurité des patients.

Le recueil de données a porté sur le déroulement des séances de traitement. L'objectif était de relever tout écart par rapport à une situation nominale et les stratégies mises en jeu par les manipulatrices pour gérer ces écarts. La définition et le périmètre d'une situation nominale relèvent d'un choix méthodologique et seront présentés ci-dessous. Afin de faciliter le recueil, une grille d'observations a été construite. Les données ont été recueillies par la méthode « papier-crayon ».

Au total, 138 séances de traitement ont été observées (97 dans le terrain A et 41 dans le terrain B), correspondant à environ 35 heures de recueil.

\section{2.- Méthodes d'analyse de données}

L'unité d'analyse considérée pour traiter les données étant la séance de traitement, une première catégorisation consiste à distinguer les séances à déroulement nominal et non nominal.

\section{Séances à déroulement nominal}

Il s'agit des séances réalisées sans que les manipulatrices aient besoin de réaliser une tâche non prévue, comme par exemple la recherche d'informations ou la correction d'une erreur détectée. Dans ce type de séance les huit conditions suivantes sont remplies : a) le dossier papier est disponible au poste, complètement et correctement rempli avant l'arrivée du patient; b) le dossier électronique est débloqué, prêt à emploi et correspond aux informations trouvées dans le dossier papier; c) tous les accessoires et appareillages sont disponibles; d) le patient est coopératif; e) les paramètres prescrits sont clairs et réalisables en poste de traitement; f) la séance est réalisée, enregistrée dans le logiciel et notée sur le dossier papier; g) le personnel est présent et disponible en cas de besoin; h) le patient a reçu la bonne dose au bon endroit. Des 138 séances observées 94 appartiennent à cette catégorie.

\section{Séances à déroulement non nominal}

Il s'agit des séances qui s'écartent des conditions supra citées, et qui comprennent donc au moins une situation non nominale (SNN). Des 138 séances observées 44 (soit $32 \%$, une fréquence significative) appartiennent à cette catégorie. Vingt séances non nominales ont été observées dans le terrain $\mathrm{A}$ et 24 dans le terrain B.

Les séances à déroulement non nominal présentent un nombre variable de situations non nominales. Les catégories de séances (séances à $0,1,2$, etc. $\mathrm{SNN}$ ) et le nombre de séances appartenant à ces 
catégories sont présentés dans le tableau 3. Au total 59 situations non nominales ont été observées, soit 24 dans le terrain A et 35 dans le terrain B.

\begin{tabular}{|c|c|c|}
\hline $\begin{array}{c}\text { Nombre de SNN par séance à } \\
\text { déroulement non nominal }\end{array}$ & Nombre de séances correspondant & $\begin{array}{c}\text { Total de SNN } \\
\text { (SNN x séance) }\end{array}$ \\
\hline 0 & 94 & 0 \\
\hline 1 & 32 & 32 \\
\hline 2 & 10 & 20 \\
\hline 3 & 1 & 3 \\
\hline 4 & 1 & 4 \\
\hline$\Sigma$ & $\mathbf{1 3 8}$ & $\mathbf{5 9}$ \\
\hline
\end{tabular}

Tableau 3: Distribution des situations non nominales dans les séances observées

Table 3 : Distribution of not-nominal situations in observed sessions

Les situations non nominales n'étant pas de même nature, une deuxième catégorisation avait pour but de coder les grands types de situations observés (cf. tableau 4). Ces catégories sont exclusives, mais les situations non nominales qui en font partie ne sont pas indépendantes dans le sens où une situation non nominale de départ peut avoir une conséquence qui engendrera une deuxième situation non nominale. Par exemple, le physicien est absent (SNN - indisponibilité du personnel) donc le dossier du patient ne sera pas prêt à temps (SNN - incomplétudes). Il existe donc des SNN « en cascade ».

Une analyse statistique descriptive a été effectuée sur l'ensemble de SNN à travers le coefficient de contingence de Cramer ( $\mathrm{V}^{2}$ de Cramer). Il permet d'établir le degré de liaison entre deux variables catégorisées (ici, terrain et type de SNN) en comparant les effectifs observés et théoriques pour chacune d'entre elles (Corroyer \& Wolff, 2003). Les résultats de cette analyse ont permis de conclure qu'il n'existe pas de liaison forte entre le terrain et le type de $\mathrm{SNN}$ observée $\left(\mathrm{V}^{2}=0.05\right.$; liaison intermédiaire).

\begin{tabular}{|c|l|c|c|c|}
\hline Types de SNN & \multicolumn{1}{|c|}{ Exemples } & $\begin{array}{c}\text { Terrain A } \\
\text { (n) }\end{array}$ & $\begin{array}{c}\text { Terrain B } \\
\text { (n) }\end{array}$ & I \\
\hline Incomplétudes & $\begin{array}{l}\text { Le dossier du patient arrive au poste sans } \\
\text { les signatures obligatoires de validation du } \\
\text { traitement. } \\
\text { Les accessoires ne sont pas disponibles au poste }\end{array}$ & 13 & 14 & 27 \\
\hline $\begin{array}{c}\text { Erreurs commises } \\
\text { au poste de } \\
\text { traitement par les } \\
\text { acteurs observés }\end{array}$ & $\begin{array}{l}\text { La manipulatrice oublie de mettre en place un } \\
\text { accessoire de traitement. } \\
\text { La manipulatrice oublie de mettre le dossier à } \\
\text { jour }\end{array}$ & 4 & 7 & 11 \\
\hline Ambiguïtés & $\begin{array}{l}\text { Les données des différents supports sont } \\
\text { contradictoires }\end{array}$ & 5 & 6 & 11 \\
\hline $\begin{array}{c}\text { Patients peu } \\
\text { coopératifs }\end{array}$ & $\begin{array}{l}\text { Le patient bouge pendant l'irradiation } \\
\text { Le patient ne garde pas les marques de repères } \\
\text { corporels utiles au placement }\end{array}$ & 0 & 2 & 2 \\
\hline $\begin{array}{c}\text { Indisponibilité du } \\
\text { personnel }\end{array}$ & $\begin{array}{l}\text { Absentéisme } \\
\text { Surcharge de travail }\end{array}$ & 1 & 3 & 4 \\
\hline $\begin{array}{c}\text { Indisponibilité du } \\
\text { matériel }\end{array}$ & L'appareil d'imagerie est en panne & 1 & 3 & 4 \\
\hline \multicolumn{1}{|c|}{$\Sigma$} & $\mathbf{2 4}$ & $\mathbf{3 5}$ & $\mathbf{5 9}$ \\
\hline
\end{tabular}

Tableau 4: Type de situations non nominales observées

Table 4 : Type of not-nominal situations observed 
La dernière phase d'analyse a consisté à décrire les différents types de régulations réalisées par les manipulatrices face aux 59 situations non nominales observées. La gestion des SNN par les manipulatrices - comme un moyen de gérer le conflit de buts - est présentée par la suite.

\section{5.- La gestion des situations non nominales comme gestion du conflit des buts « produire la santé » et « produire la sécurité » des patients}

La période avant le début d'une séance en radiothérapie correspond à une analyse de conformité afin de s'assurer qu'il n'existe pas d'écart par rapport à une situation nominale (cf. définition au point 4.2). Si toutes les conditions qui définissent une situation nominale sont remplies, le traitement est réalisé. Il n'y a pas de conflit entre les objectifs de «production de la santé » et «production de la sécurité des patients ». Le patient a eu son traitement sans que la manipulatrice ait eu à privilégier l'un de ces deux objectifs.

En cas de survenue d'une situation non nominale, un conflit entre ces deux objectifs apparait:

- D’une part si la séance est réalisée elle ne le sera pas dans les conditions prescrites. L'objectif de produire la santé du patient est atteint au détriment de l'objectif de produire la sécurité.

- D'autre part, si la séance est annulée, la situation inverse se présente, c'est-à-dire l'objectif de produire la sécurité du patient est atteint au détriment de l'objectif de produire la santé de celuici.

Dans les deux cas les manipulatrices prennent un risque: risque pour le patient mais aussi pour elles. On peut leur reprocher d'avoir administré le traitement dans des conditions non nominales ou de ne pas l'avoir fait alors qu'il était nécessaire.

Il est important de souligner ici que la prescription formelle (règles écrites) est réduite en radiothérapie. Ceci a été soulevé comme un facteur de risque dans d'autres études (ASN 2007; MeaH 2007). Dans ce contexte on peut s'attendre à la construction de règles implicites, au niveau individuel et collectif (Nascimento \& Falzon 2008b). Les régulations opérées par les manipulatrices s'inscrivent ainsi dans un contexte de prescription floue, fortement informel.

Dans un premier temps, afin de gérer les conflits de buts, les manipulatrices vont tenter de récupérer la situation non nominale, en la rendant conforme à un état souhaité/nominal. Dans la majorité des cas elles arrivent à gérer les situations sans avoir à privilégier un des buts d'action. Parfois la récupération de la situation est possible en local, c'est-à-dire par les manipulatrices elles-mêmes en poste d'administration. Cependant, dans certaines situations, l'appel à un membre extérieur (un radiothérapeute, un physicien médical, un dosimétriste, un technicien) est indispensable pour la gestion de la situation non nominale et ainsi pour la poursuite des deux objectifs d'actions des manipulatrices. Ces comportements sont similaires dans les deux terrains observés.

Quand ces types de régulations ne sont pas possibles, par exemple en cas d'indisponibilité d'un membre extérieur, la gestion du conflit se fera par le développement d'un compromis entre le risque pour le patient et le bénéfice pour le patient. Ceci conduit à des décisions de sacrifice, c'est-à-dire à privilégier un des deux objectifs d'action: produire la santé ou produire la sécurité des patients. Ce compromis est fortement fondé sur la connaissance des collègues, sur la satisfaction du patient et sur la phase du traitement. Des différences entre les comportements des manipulatrices des deux terrains vont apparaître.

Le diagramme d'activité présenté ci-dessous synthétise l'ensemble des actions des manipulatrices afin de gérer le conflit de buts rencontrés dans leurs pratiques. 


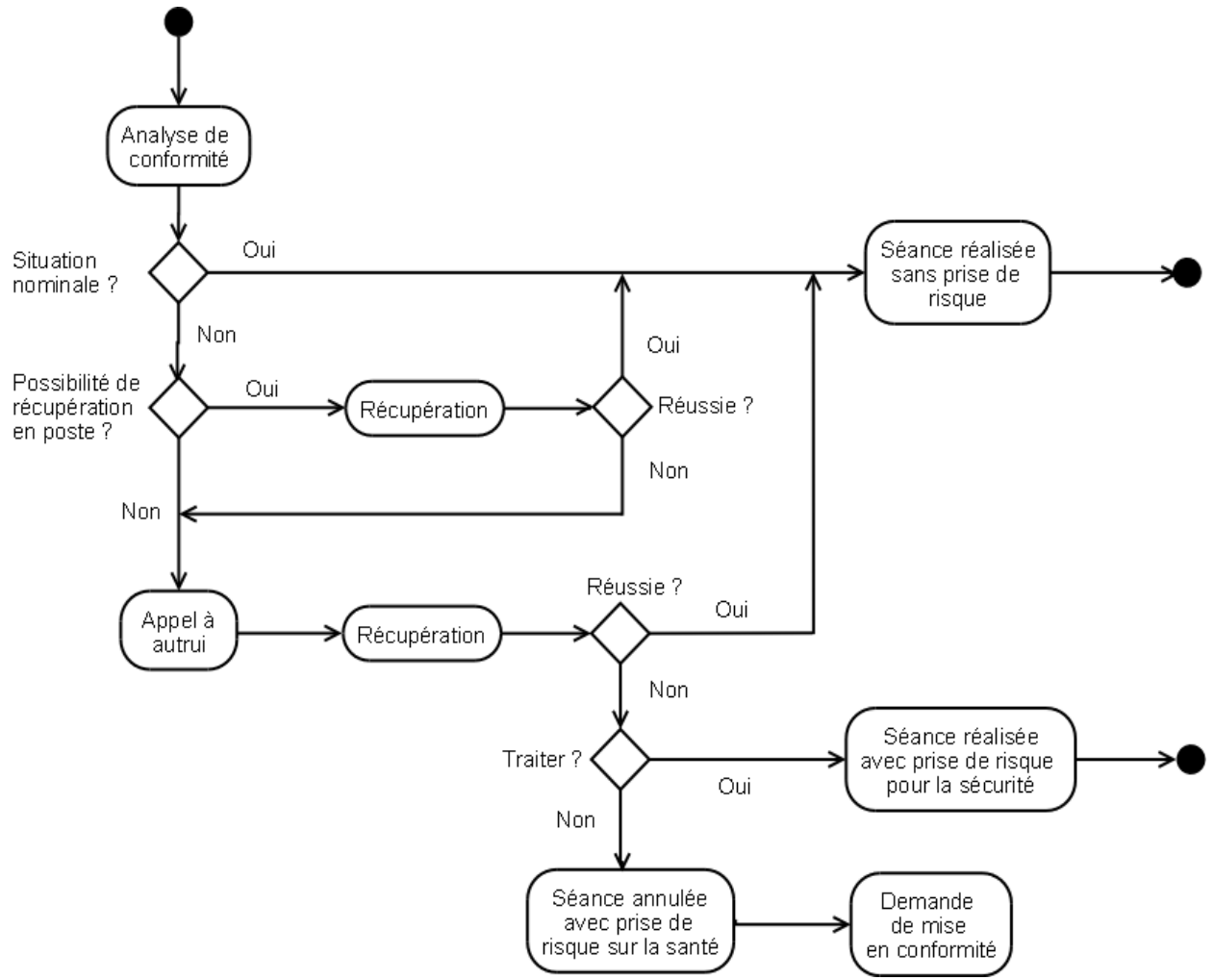

Figure 1: La gestion du conflit entre les buts «produire la santé » et « produire la sécurité »

Figure 1: Managing conflicts between «ensuring health» and «ensuring safety

\section{1.- Les régulations comme un moyen d'éviter le compromis dans le risque}

\subsection{1.- Les régulations possibles en local}

Les SNN concernant les erreurs commises au poste de traitement (11 cas), les patients peu coopératifs ( 2 cas), 5 cas d'ambiguïtés et 2 cas d'incomplétudes survenues dans le terrain $B$ ont été gérées en local par les manipulatrices.

La gestion des erreurs commises au poste prend une place marginale dans les activités des manipulatrices. Ceci est dû au faible nombre d'erreurs produites au poste (11/59) mais surtout à la facilité et rapidité de récupération après détection. Toutes les erreurs observées dans les deux terrains ont été détectées, soit par le système informatique soit par l'acteur de l'oubli soit par l'équipe du poste. Un oubli a été détecté par une patiente. Prenons deux exemples de détection et récupération des erreurs en poste de traitement.

Cas 1: Le système informatique détecte les oublis de mise en place de certains accessoires avant la réalisation de l'irradiation. L'absence d'accessoire est signalée sur le pupitre de commande de l'accélérateur et la manipulatrice corrige l'erreur, mettant en place l'accessoire oublié. Cas 2: Un dossier non mis à jour a été détecté par l'équipe du poste. Le professionnel qui détecte l'erreur signale aux collègues à la fois l'écart et sa récupération, l'objectif étant la prise de conscience collective de la survenue de l'oubli et de sa gestion.

Vous avez oublié de noter la séance de Mme X d'hier. Je l'ai fait. Manipulatrice 1, terrain B

La gestion des patients peu coopératifs s'est produite deux fois durant les observations dans le terrain B. À une reprise, durant l'irradiation, les manipulatrices détectent - via les caméras de surveillance 
- des mouvements réalisés par le patient. Les manipulatrices essaient d'expliquer au patient - par l'interphone - l'importance de rester immobile durant l'administration de l'irradiation. Le patient ne respecte pas cette consigne et, entre deux faisceaux, une des manipulatrices revient en salle d'irradiation pour essayer de le convaincre. Le deuxième cas concerne une patiente qui a fait disparaître les marques de repères pour le placement. Les régulations des manipulatrices face à ce cas consistent d'une part à informer la patiente - comme pour le cas précédent - de l'importance des traits de repérages pour l'irradiation de l'endroit exact, d'autre part à réaliser à nouveau les traits de repérages. Dans les deux exemples les actions des manipulatrices ont pour objectif de répondre aux deux buts de production des soins, en s'assurant que le traitement sera délivré à l'endroit prescrit.

Quant à la gestion des incomplétudes, 12 cas sur 27 ont pu être résolus par les manipulatrices soit parce que l'incomplétude est issue de manipulatrices qui sont extérieures au poste observé ( 2 cas, terrain B) - celles qui sont au simulateur, par exemple; soit parce que la gestion de l'incomplétude issue d'autres métiers n'empêche pas l'action des manipulatrices (10 cas qui seront discutés en point 5.2). Dans le premier cas la gestion est faite à partir de connaissances techniques propres au métier: les traits de repères pour le placement du patient qui auraient dû être faits au simulateur sont absents, les manipulatrices le redessinent sur poste de traitement. Ceci a posé des problèmes par rapport à la gestion du temps, car ce travail n'était pas prévu et est long à réaliser (20 minutes environ), et par rapport à la fiabilité du placement. Toute la préparation du traitement se fait en fonction des placements qui sont réalisés lors de l'étape sur simulateur. « On est en train de réaliser une simulation sur table, c'est-à-dire tout ce qui aurait dû être fait au simulateur, on le fait ici », explique la manipulatrice.

Le dernier type de gestion réalisée en local comprend les ambiguïtés (5 cas, terrain B) qui peuvent être récupérées sans intervention de membres extérieurs au poste. Il s'agit des données contradictoires sur les différents supports. Une des gestions réalisées est présentée dans l'exemple suivant. En vérifiant un dossier papier la manipulatrice détecte que la distance source-peau (DSP) prescrite est de $92,5 \mathrm{~cm}$ et qu'elle devrait être de $95,2 \mathrm{~cm}$. Ce type de détection montre l'expérience du professionnel par rapport à la technique utilisée dans le sens où il sait que la DSP standard est de $95,2 \mathrm{~cm}$.

L'inquiétude a été ensuite de savoir si le traitement a été réalisé avec la «bonne » ou la « mauvaise » DSP. La stratégie de la manipulatrice consistera à gagner du temps et à ne pas déranger un collègue : elle regarde sur le dossier informatisé de délivrance de la dose plutôt que d'appeler un physicien. Après avoir confirmé que le dossier indique la DSP standard, la manipulatrice réalise la séance.

Peut-être le traitement s'est fait correctement et c'est juste sur le dossier papier que ce n'est pas bon. Avant d'appeler un physicien on regarde si sur le logiciel c'est bon Manipulatrice 2, terrain B

En résumé, 20 des 59 situations non nominales ont été gérées au niveau du poste de traitement, sans intervention d'acteurs extérieurs. Ceci correspond à la gestion de $16 \%$ des SNN observées dans le terrain A et de $46 \%$ des SNN observées dans le terrain B. Cette différence est importante et montre que dans le terrain A seules les erreurs commises en local ont été récupérées en local. Les manipulatrices récupèrent leurs propres erreurs tandis que pour les autres types d'écarts le collectif est engagé. Ceci peut être expliqué par le nombre plus important de professionnels dans le terrain A.

De manière globale, les régulations mises en œuvre par les manipulatrices des deux terrains ont permis l'administration des soins en accord avec leurs deux objectifs d'action, évitant des arbitrages.

\subsection{2- Les régulations opérées en mobilisant le collectif}

Certaines situations non nominales n'ont pas pu être complètement gérées par les manipulatrices. Celles-ci détectent les écarts, mais leurs récupérations dépendent d'autres métiers. Il s'agit des SNN concernant 6 cas d'ambiguïtés, 2 cas de pannes totales d'appareil de traitement et 15 cas d'incomplétudes.

Dans le terrain A la situation d'appel au collectif face à une ambiguïté a été observée 5 fois et dans le 
terrain $\mathrm{B}$ ceci est arrivé 1 seule fois. Pour ne pas prendre de risque, l'appel à autrui a été systématique dans ce type d'écart.

L'exemple suivant décrit la gestion de ce type d'ambiguïté dans le terrain A. Deux manipulatrices placent une patiente pour un traitement d'un cancer du sein. Elles se rendent compte que le champ d'irradiation virtuellement projeté sur la patiente présente une fuite vers un autre champ devant être irradié (l'auxiliaire). Les manipulatrices ont un doute sur la taille du champ et décident ensemble d'appeler le médecin responsable de la patiente: «On ne prend pas de risque », dit une manipulatrice à sa collègue. Étant mis au courant du cas, le médecin demande le nombre de centimètres de fuite vers le champ auxiliaire. La manipulatrice lui répond qu'il s'agit d'environ un centimètre de fuite. Le médecin considère que c'est un écart important et décide de le vérifier sur place. Le champ projeté sur la patiente ne correspond effectivement pas à la prescription, c'est-à-dire au souhait du médecin. Celui-ci redessine sur la patiente un champ qui lui convient mieux. Une manipulatrice rectifie la prescription sur le dossier de la patiente en écrivant le message suivant: " attention, se mettre sur les marques vertes [et non bleues] pour le champ ». Le traitement est réalisé comme souhaité par le médecin.

Si la séance avait été réalisée avec la prescription erronée, la partie du champ auxiliaire contenant la fuite aurait été irradiée deux fois, ce qui constitue un surdosage. La sécurité de la patiente a été assurée par les manipulatrices qui en détectant un écart ont choisi de ne pas décider elles-mêmes si cet écart était important ou négligeable.

Le deuxième type de régulations faisant appel au collectif concerne la gestion des pannes. Deux pannes totales d'appareils ont été observées dans le terrain A. Les manipulatrices étaient dans l'impossibilité de réaliser les traitements. Elles ont fait appel à un technicien qui a résolu le problème immédiatement. Cet événement a été sans conséquence pour l'administration des soins en sécurité.

Le troisième type de SNN gérées grâce à l'appel au collectif concerne des incomplétudes. Dans 15 des 27 situations d'incomplétudes observées ( 5 dans le terrain A et 10 dans le terrain B), les manipulatrices étaient dans l'impossibilité de délivrer les soins. Dans ce genre de situation, l'ensemble des moyens nécessaires à la réalisation du traitement n'était pas réuni au poste de traitement du fait de l'absence de dossiers de traitement ou de moyens de contention des patients. L'absence du dossier de traitement était le cas le plus fréquemment observé (13 fois). Les dossiers étaient soit disponibles au poste de traitement en version papier mais indisponibles sur le logiciel soit complètement indisponibles. Le cas d'un dossier disponible uniquement sur logiciel n'a pas été observé.

Afin de répondre au but de «produire la santé » des patients, lors de la détection d'un retard dans l'arrivée du dossier au poste par rapport à l'arrivée du patient, une des manipulatrices se charge d'appeler le physicien ou le dosimétriste ou d'aller directement en salle de préparation de la dosimétrie pour rechercher le dossier en question. Dans le terrain A, les manipulatrices vont plutôt aller chercher le dossier et dans le terrain B elles auront tendance à appeler et demander que les physiciens ou dosimétristes leur apportent le dossier s'il est prêt. Ces comportements sont dictés par le nombre de manipulatrices au poste, les manipulatrices du terrain B étant moins nombreuses et ne souhaitant pas laisser leurs collègues seules pour aller chercher un dossier. Les régulations ici observées consistent à rechercher un autre professionnel. Les dossiers papiers et informatiques sont indisponibles et la manipulatrice est dans l'incapacité de réaliser le soin.

Néanmoins, ces régulations ont des limites. Dans certains cas les professionnels recherchés sont disponibles et compléteront le dossier ou communiqueront un dossier déjà prêt. Cependant dans d'autres cas, les professionnels appelés ne seront pas disponibles dans l'immédiat. Ceci constitue une situation non nominale qui va conduire à une autre situation non nominale de deuxième niveau qui est l'annulation de la séance de traitement. Ce cas a été observé quatre fois et sera discuté en point 5.2 .

En résumé, 23 des 59 situations nominales ont été gérées par les manipulatrices grâce à l'intervention d'acteurs extérieurs. Ceci correspond à la gestion de $50 \%$ des SNN observées dans le terrain A et de 
$32 \%$ des SNN observées dans le terrain B.

Encore une fois, les régulations mises en œuvre par les manipulatrices ont permis l'administration des soins en accord avec leurs deux objectifs d'action. Ces régulations visent en réalité à éviter le compromis dans le risque, c'est-à-dire à éviter des arbitrages qui vont favoriser seulement l'un des objectifs de production de la santé et de production de la sécurité des patients.

Au total 43 des 59 SNN qui ont demandé une gestion de la part des manipulatrices ont eu leurs récupérations réussies en termes d'évitement du compromis entre les buts d'actions. Ceci a été possible grâce aux moyens de récupérations disponibles en local, c'est-à-dire par les manipulatrices elles-mêmes ou grâce à l'intervention d'un membre extérieur au poste. Dans les deux cas les séances ont été réalisées sans prise de risques, ni pour la santé ni pour la sécurité des patients.

Il s'agit ici de régulations d'un premier niveau, qui impliquent des choix et des décisions (traiter le cas en local ou appeler un physicien/médecin, par exemple) visant à éviter les dilemmes/décisions de sacrifice. En cas d'échec, un deuxième niveau de régulations a lieu et concerne les décisions de sacrifice devant un conflit de buts.

\section{2- Le compromis dans le risque : la priorité donnée à l'un des objectifs d'action}

Les résultats présentés jusqu'ici montrent que les manipulatrices arrivent à faire face à la majorité des situations non nominales à travers des régulations fondées sur des habilités propres à leurs métiers ou sur celles des membres du collectif. Le conflit de buts est géré par l'évitement des décisions de sacrifice entre les deux objectifs d'action.

Dans 16 cas, ces types de régulation n'ont pas pu éviter le compromis entre "produire la santé »et « produire la sécurité » des patients. Ceci est lié à la nature des situations non nominales. Il s'agit des écarts produits en amont de la chaîne (incomplétudes) et des pannes partielles d'appareil qui n'ont pas pu être récupérés par l'intervention des collègues.

Parmi le premier type d'écart - les incomplétudes - les manipulatrices ont fait face à deux types de situations :

- Situation 1: des dossiers n'étaient pas prêts avant l'arrivée des patients et les professionnels responsables pour leur préparation n'étaient pas disponibles pour résoudre le problème dans l'immédiat. Ils étaient soit absents soit occupés par une tâche plus urgente. Deux situations non nominales s'enchaînent ici: le dossier n'est pas prêt et le personnel est indisponible. Ce cas a été observé quatre fois, soit une fois dans le terrain A et trois fois dans le terrain B. Face à ces situations non nominales, les manipulatrices n'ont pas eu à prendre une décision: l'indisponibilité des dossiers (papier et informatique) ajoutée à l'indisponibilité des professionnels qui devraient les préparer a empêché l'administration des soins. La séance d'irradiation a été annulée et ceci a constitué une troisième situation non nominale. Le but de production de la santé des patients n'est pas atteint, mais ceci n'est pas le résultat d'une prise de décision de la part des manipulatrices;

- Situation 2: des dossiers sont disponibles au poste, sous format électronique et papier, mais les accords des radiothérapeutes - via signature - n'y sont pas présents. Cette situation a été observée à 10 reprises, soit 8 dans le terrain $\mathrm{A}$ et 2 dans le terrain $\mathrm{B}$. Les manipulatrices ont la possibilité d'administrer les soins malgré l'absence des validations, ce qui laisse des marges pour des régulations en local. Dans ce sens, et à l'inverse de la situation 1, les manipulatrices vont prendre une décision. Elle consistera à traiter ou pas un patient dont le plan de traitement n'a pas été validé par un radiothérapeute. Cette décision concerne en réalité la recherche d'un compromis entre les objectifs de production de la santé et de la sécurité. En point 5.2.1 nous reviendrons sur les facteurs à l'origine des décisions prises par les manipulatrices.

Concernant le deuxième type d'écart - les pannes du système d'imagerie embarqué non récupérée par les techniciens - les possibilités de régulations par les manipulatrices sont similaires à la situa- 
tion 2 présentée ci-dessus. L'administration de soins est possible car la panne concerne seulement le système d'imagerie, l'appareil d'administration de la dose fonctionnant normalement. Le cas d'une panne d'imagerie a été observé 2 fois dans le terrain B. Pour la gestion de ce type de panne, les manipulatrices ont dû faire un choix, c'est-à-dire qu'elles ont mis en œuvre des stratégies visant à atteindre l'un des deux buts d'actions: traiter le patient sans être sûres de son placement correct et ainsi produire la santé ou annuler la séance pour ne pas prendre le risque d'irradier une zone non désirée et produire ainsi la sécurité des patients. Au point suivant nous allons revenir sur les facteurs qui permettent cette prise de décision.

Pour la gestion de ces 12 situations non nominales (10 incomplétudes +2 pannes partielles), les manipulatrices ont dû faire un compromis dans le risque entre les deux buts d'actions « produire la santé » ou « produire la sécurité » des patients. Les arbitrages observés se construisent à partir des logiques qui prennent en compte l'évaluation des risques et des bénéfices pour les patients. Ils sont fondés sur la connaissance du fonctionnement du collectif, voire des individus, sur la phase du traitement (début ou en cours), sur la satisfaction des patients et sur la construction de la culture collective de sécurité.

\subsection{1.- Critères de décision}

\section{- La phase de traitement}

Des images de contrôle de positionnement du patient en table de traitement sont réalisées lors des séances 0,1 et 2, puis une fois par semaine. L'objectif est de vérifier la conformité entre le positionnement prévu au simulateur (donc celui sur lequel le traitement a été planifié) et le positionnement réel du patient en poste de traitement. C'est un moyen de s'assurer que le patient recevra la dose prescrite à l'endroit prescrit, sachant qu'un décalage de $1 \mathrm{~cm}$ du volume tumoral est considéré comme une erreur en radiothérapie.

Durant nos observations, l'appareil d'imagerie qui sert à ce contrôle, appelé portal, est tombé en panne à 2 reprises lors de l'ouverture du service. Les régulations des manipulatrices face aux pannes ont consisté à appeler la dosimétriste qui à son tour a contacté le constructeur de l'appareil. Un technicien lui répond qu'il arrivera pour réparer la panne dans la journée. Pendant cette attente, les manipulatrices décident de traiter les patients qui sont en cours de traitement et d'annuler les séances de ceux qui débutent la radiothérapie.

Les patients qui sont en cours de traitement doivent avoir une image de contrôle une fois par semaine. Normalement ceci est réalisé tous les lundis - jour où l'appareil d'imagerie est tombé en panne. Néanmoins, les manipulatrices considèrent que le risque de ne pas traiter les patients en cours de traitement est plus important que le bénéfice d'annuler leurs séances. L'objectif de production de la santé a été donc privilégié pour ces patients.

Cependant, pour les patients qui débutent un traitement, les manipulatrices préfèrent les appeler pour annuler leurs séances - et les rendre mécontents du service - que prendre le risque de réaliser le traitement sans être certaines de l'administration d'une dose correcte à l'endroit prescrit. Elles ne connaissent pas encore ces patients et ne savent pas comment ils se positionnent sur la table de traitement. Dans ce cas, les manipulatrices vont préférer privilégier le but de sécurité des patients et ne pas réaliser la séance de traitement.

\section{- La connaissance des collègues}

L'arrivée d'un dossier au poste de traitement sans la validation du radiothérapeute a été observée 10 fois. Les régulations des manipulatrices face à 8 de ces situations non nominales ont été fondées sur la connaissance de l'autre.

Un radiothérapeute peut décider de ne pas valider ses traitements quand il s'agit de cas très protocolisés, et par conséquent considérés comme simples. Pour gagner du temps, il ne valide le traitement qu'à la première consultation hebdomadaire avec le patient, c'est-à-dire une semaine après le début 
du traitement. Les stratégies des manipulatrices seront fondées sur la connaissance du comportement des radiothérapeutes face aux cas protocolisés/simples. Elles considèrent avoir un accord implicite pour la réalisation de ce type de traitement.

Un médecin qui a toujours les mêmes traitements, les mêmes modifications, c'est rare qu'il signe les dosimétries, il faut presque qu'on lui prenne par la main pour qu'il les signe. Manipulatrice 4, terrain A

Les règles d'action des manipulatrices sont également guidées par le fonctionnement du binôme physicien-radiothérapeute. Dans ce sens, pour les manipulatrices, la validation du dossier par un physicien peut sous-entendre l'accord du radiothérapeute pour la réalisation du traitement.

Les dosimétries de la physicienne $X$ vont rarement être signées par un médecin, parce qu'elle est à un an de la retraite, elle connaît son travail sur le bout des doigts et ils [les médecins] lui font confiance. Manipulatrice 5, terrain A

La manipulatrice connaît le « contrat de confiance » construit entre les radiothérapeutes et la physicienne X. Les radiothérapeutes ne valident pas les traitements conçus par cette physicienne, car ils sont certains qu'elle maîtrise son travail. Ainsi, les traitements conçus par la physicienne X peuvent être réalisés en l'absence de validation formelle des radiothérapeutes.

En effet, les adaptations opérées par les manipulatrices se caractérisent par la construction de règles d'actions qui vont à l'encontre des procédures formelles, mais qui répondent aux attentes de l'organisation et à l'objectif de « produire la santé » des patients. Ce qui prime dans ce choix c'est la production des soins, donc la réalisation du traitement. Elles prennent ici un risque raisonné visant le bénéfice pour le patient. Elles considèrent que le risque pris par la réalisation de la séance même avec un dossier non validé est moindre que le risque du patient ne pas avoir son traitement du jour.

\section{- La construction de la culture collective de sécurité}

Les régulations mises en œuvre par les manipulatrices devant un dossier non validé par le radiothérapeute ont été fondées également sur l'intérêt de construire une culture collective de sécurité à travers la prévention d'un comportement non nominal chez ces professionnels.

On pourrait traiter, c'est simple. Mais si l'on fait ça, ils [les médecins] prennent trop l'habitude de ne pas les valider [les dossiers] systématiquement » Manipulatrice 3, terrain B

Cette règle d'action répond à la procédure, mais elle est aussi fondée sur la connaissance du fonctionnement du collectif et au-delà elle porte un message au collectif. On voit ici un changement d'échelle: le choix ne se joue plus entre les deux buts vis-à-vis du patient présent, mais entre intérêt du patient présent et intérêt des patients futurs, à travers le développement d'une meilleure culture de sécurité au sein de l'équipe. Dans ce sens le risque de ne pas traiter le patient est contrebalancé par le bénéfice de contribuer à l'augmentation du niveau de culture collective de sécurité.

Dans ces situations les manipulatrices considèrent que le risque de réaliser le traitement avec un dossier non validé par un radiothérapeute est plus important que le bénéfice escompté par la réalisation de la séance. L'objectif de " produire sécurité » des patients a été privilégié au détriment de l'objectif de «produire la santé » car le traitement du jour n'a pas été réalisé.

\section{- La satisfaction des patients}

Face à un dossier non validé par un radiothérapeute, les stratégies d'action des manipulatrices ont également été fondées - au-delà du choix raisonné pour privilégier la construction collective de la sécurité discuté plus haut - sur la « satisfaction » des patients.

Il s'agissait de la séance 1, c'est-à-dire de la première séance d'irradiation. La veille (séance 0) les images de contrôle sur le positionnement ont été réalisées. Ces images n'ont pas été validées par le radiothérapeute et il est absent à ce jour. Les régulations des manipulatrices ont consisté à réaliser à nouveau les images de contrôle afin de donner l'impression aux patients qu'ils « ne se sont pas déplacés pour rien ». Ces régulations visent à atténuer les défaillances de l'organisation, sans pour 
autant violer la procédure. L'objectif de production de la santé n'est pas atteint car les patients n'ont pas leurs séances du jour, mais ils n'auront pas l'impression que le service n'a pas été réalisé.

\subsection{2- Comparaison inter terrains}

La recherche d'un compromis entre les objectifs de production de la santé et production de la sécurité des patients a différé selon le terrain observé. Face à la présence en poste de traitement d'un dossier non signé (validé) par le radiothérapeute, il a été observé que des manipulatrices de terrains différents ont des raisons différentes de respecter ou ne pas respecter la procédure réglementaire qui consiste à «ne pas traiter un patient sans signature du médecin sur le dossier ».

Dans le terrain A, l'arrivée d'un dossier au poste de traitement sans la validation du radiothérapeute a été observée 8 fois. Le traitement a été réalisé systématiquement. En réalité, cette prise de décision pour la réalisation du traitement, malgré l'absence d'accord final du radiothérapeute, est fondée sur la connaissance du fonctionnement du collectif. Les manipulatrices répondent à la tâche attendue ou tolérée par l'organisation. Celle-ci consiste à traiter sans la validation du radiothérapeute - et ainsi privilégier la production de la santé des patients - sous réserve qu'il s'agisse d'un cas simple et/ou que le dossier ait été validé par un physicien. La validation par un physicien sous-entend en réalité l'accord verbal ou inconditionnel du radiothérapeute.

Dans le terrain B, l'arrivée d'un dossier au poste de traitement sans la signature du médecin a été observée 2 fois. À ces 2 occasions les séances d'irradiation ont été annulées, cependant les patients ont eu le sentiment d'avoir été pris en charge. Les stratégies d'action des manipulatrices sont fondées d'une part sur la « satisfaction » du patient et d'autre part sur la prévention d'un comportement non nominal chez les radiothérapeutes.

\section{6.- La gestion des risques par les manipulatrices en radiothérapie}

Les situations non nominales observées dans le domaine de la radiothérapie peuvent se caractériser comme les « gaps » étudiés par Cook, Render et Woods (2000) chez les opérateurs de bout de ligne dans le domaine hospitalier: il s'agit de situations qui se traduisent par un manque d'information ou par un moment d'interruption et qui peuvent, si non récupérées, empêcher l'administration de soins. Ces auteurs soulignent que les gaps sont très fréquents en milieu de soins mais qu'ils conduisent rarement à l'accident parce que les professionnels de dernière ligne sont aptes à les récupérer.

De la même manière, nos observations ont pu mettre en évidence l'importance des manipulatrices en radiothérapie dans la gestion de la sécurité en bout de chaîne. Des 59 situations non nominales observées dans nos terrains de recherche, à notre connaissance aucune n'a conduit à un écart de dose. La gestion des situations non nominales a consisté dans un premier temps à éviter de privilégier, face à un conflit de buts, un des deux buts d'action. Les situations ont été récupérées soit par les manipulatrices elles-mêmes soit à travers la mobilisation du collectif.

Dans un deuxième temps, quand le conflit n'a pas pu être évité, la gestion des situations non nominales a conduit les manipulatrices à faire des décisions de sacrifice entre santé et sécurité des patients. Ces régulations sont proches de celles observées par Gomes, Woods, Carvalho, Huber et Borges (2009, op. cit.) chez les pilotes d'hélicoptère. Elles consistent à sacrifier un des buts en analysant les risques et les bénéfices escomptés par la sécurité/productivité.

Dans ces types de cas, la gestion des situations a engendré des situations non nominales d'un deuxième niveau: soit l'annulation d'une séance prévue, soit la réalisation d'une séance avec un moindre niveau de sécurité. D'un côté, il a été observé un «glissement » par rapport à la réglementation dans un but de produire les soins et ainsi la santé des patients. Le bénéfice escompté a été considéré comme étant plus important que le risque d'erreur.

D'un autre côté, et à l'inverse, les opérateurs font en sorte d'empêcher ces « glissements » à travers 
une forme de contrôle dans lequel le comportement non nominal est signalé à une personne qui peut prendre des décisions pour l'éviter: c'est le principe du whistle-blowing (Miceli, Near, \& Dworkin, 2008). Ce type de systèmes d'alerte a été également observé par King (2001) en milieu hospitalier. Ils visent la prévention des situations non nominales. Dans le cas des manipulatrices qui n'ont réalisé les traitements sans les validations médicales, ceci sert de signe d'alerte pour le médecin en question et pour l'organisation. Les préjudices issus du fait de l'annulation des séances sont contrebalancés par les bénéfices d'assurer la sécurité des patients d'une part, et la prise de conscience collective des défaillances d'autre part.

En réalité les différences observées lors de la prise des décisions de sacrifice mettent en évidence l'existence de cultures de sécurité, qui sont propres à chaque établissement, et qui sont construites au fil des temps sur la base d'un système flexible qui concilie la sécurité par les règles et l'adaptation aux cas particuliers. L'objectif des opérateurs est de « rester maîtres de la situation, c'est-à-dire être dans les marges de sécurité pour le niveau de performance visée » (Amalberti, 2007, p. 81). C'est la notion de «domaine acceptable » qui vient, selon Amalberti (ibid.), remplacer celle d'une « ligne rigide à suivre ». Le domaine acceptable doit néanmoins être défini et discuté par l'ensemble des professionnels afin de mieux délimiter les frontières de la fiabilité en radiothérapie.

\section{7.- La mise en visibilité de l'activité : une ressource pour le développement d'une culture collective de sécurité}

Ces résultats ont fait objet d'une restitution collective avec les professionnels de l'établissement A. Le débat collectif qui a suivi la restitution indique un manque de visibilité des activités réalisées en bout de chaîne. Ce retour vers les professionnels s'est montré un outil puissant pour le développement de la culture collective de sécurité, et ceci à deux niveaux :

\section{- Prise de conscience du rôle de chacun dans la production collective de la sécurité}

Le moment de la restitution était l'occasion de rendre visible l'activité des manipulatrices pour l'ensemble des acteurs. La plupart des professionnels n'étaient conscients ni du rôle de récupération tenu quotidiennement par les manipulatrices, ni de l'impact de leurs propres activités sur l'activité des manipulatrices en bout de chaîne. Or, d'une part, la présence de chacun dans l'activité de tous et la présence de tous dans l'activité de chacun font partie des conditions d'efficacité du travail coopératif (Darses \& Falzon, 1996) et, d'autre part, l'augmentation du niveau de culture de sécurité ne peut être effective sans une prise en compte de tous les niveaux de défaillances du système sociotechnique, y compris les contraintes réelles de l'ensemble des individus (Pronovost \& Sexton 2005).

\section{- Confrontation des pratiques et développement du collectif}

Dans un deuxième temps du débat, les professionnels justifient ce manque de conscience collective d'une part par une présence insuffisante sur les postes de traitement. En effet, les médecins, physiciens et dosimétristes ne réalisent pas leurs activités au poste de traitement et n'y interviennent que rarement. D'autre part, il a été évoqué que les lieux d'échange existants dans ce service de radiothérapie, notamment la cellule de retour d'expérience (CREX), ne favorisent pas la discussion sur les types de régulation observés au poste de traitement. La CREX, telle quelle opère actuellement, ne prend en compte que les situations d'écart de dose avéré. Ces explications offrent des marges de progrès pour les actions visant l'amélioration de la culture de sécurité, à travers la construction d'un collectif sûr.

Les espaces d'échanges au sein des équipes pourraient être adaptés, l'objectif étant que la promotion de la sécurité soit vécue plus collectivement et que chaque professionnel sache le poids de son travail dans le travail des autres. La confrontation des pratiques dans ces collectifs transverses peut s'inspirer des travaux qui proposent une pratique réflexive au travail (Pidgeon \& O'Leary, 1994; Falzon \& Teiger, 1995 ; Mollo \& Falzon, 2004) « dans laquelle l'opérateur se donne comme objet de travail sa propre activité de travail » ou celle de leurs collègues (Falzon \& Teiger, 1995). 
La mise en place de tels espaces de confrontation des pratiques aurait des bénéfices immédiats en termes de mise en visibilité de l'activité de chacun, de correction de certaines pratiques ou de création de règles d'action collectives. De manière plus générale, ces espaces, outils de développement du collectif, constitueraient des éléments d'un environnement capacitant (Falzon, 2005; Pavageau, Nascimento, \& Falzon, 2007; Falzon, Nascimento \& Pavageau, 2008; Falzon, \& Mollo, 2009).

\section{RÉFÉRENCES}

Amalberti, R. (2007). Une épée de Damoclès pour les hautes technologies. Sciences à risques. Les dossiers de la recherche, $\mathrm{n}^{\circ} 26,74-81$.

ASN (2007). Radioprotection des patients en radiothérapie externe: prévention des incidents par une approche sur les facteurs humains et organisationnels. Canevas d'inspection, Autorité de Sûreté Nucléaire, 36.

Caroly, S., \& Weill-Fassina, A. (2004). Evolutions des régulations de situations critiques au cours de la vie professionnelle dans les relations de service. Le travail humain, 67(4), 305-332.

Chapet, O., \& Mornex, F. (2006). Radiobiologie. In J. J. Mazeron, A. Maugis, C. Barret, \& F. Mornex (Eds.), Techniques d'irradiation des cancers : la radiothérapie conformationnelle (pp. 13-19). Paris: Ed. Maloine.

CIPR. (2000). Prévention des expositions accidentelles chez les patients recevant une radiothérapie (Vol. Publication 86). EDP Sciences.

Cook, R., Nemeth, C., \& Dekker, S. (2008). What went wrong at the Beaston Oncology Center? In E. Hollnagel, C. Nemeth, \& S. Dekker (Eds.), Resilience Engineering Perspectives: Remaining Sensitive to the Possibility of Failure (Vol. 1). Aldershot, England: Ashgate Studies in Resilience Engineering.

Cook, R. I., Render, M., \& Woods, D. D. (2000). Gaps in the continuity of care and progress on patient safety. British Medical Journal, 320, 791-794.

Corroyer, D., \& Wolff, M. (2003). L'analyse statistique des données en Psychologie: concepts et méthodes de base. Paris: Armand Colin.

Cosset, J.-M., \& Gourmelon. (2002). Accidents en radiothérapie: un historique. Cancer/Radiother 6(Suppl. 1), 166-170.

Darses, F., \& Falzon, P. (1996). La conception collective: une approche de l'ergonomie cognitive. In G. de Terssac, \& E. Friedberg (Eds.), Coopération et conception (pp. 123-135). Toulouse: Octarès.

Dekker, S. (2003). Failure to adapt or adaptations that fail: contrasting models on procedures and safety. Applied Ergonomics, 34(3), 233-238.

Falzon, P. (2005). Ergonomics, knowledge development and the design of enabling environments. HWWE'2005, Humanizing Work and Work Environment Conference, December 10-12 Guwahati, India (pp. 1-8).

Falzon, P., \& Mollo, V. (2009). Pour une ergonomie constructive: les conditions d'un travail capacitant. Laboreal (accepté pour publication).

Falzon, P., Nascimento, A., \& Pavageau, P. (2008). Towards models and tools for assessing the developmental quality of work. In L. Sznelwar, F. Mascia, \& U. Montedo (Eds.), Human Factors in Organizational Design and Management - IX. Sao Paulo, 19-21 mars 2008.

Falzon, P., \& Teiger, C. (1995). Construire l'activité. Performances Humaines \& Techniques, Hors Série (septembre), 34-39.

Georgin, J., \& Barret, C. (2006). Le rôle du technicien en radiothérapie. In J. J. Mazeron, A. Maugis, C. Barret, \& F. Mornex (Eds.), Techniques d'irradiation des cancers : la radiothérapie conformationnelle, (pp. 13-19). Paris: Ed. Maloine. 
Gomes, J. O., Woods, D. D., Carvalho, P. V. R., Huber, G. J., \& Borges, M. R. S. (2009). Resilience and brittleness in the offshore helicopter transportation system: The identification of constraints and sacrifice decisions in pilots' work. Reliability Engineering \& System Safety, 94(2), 311-319.

King, G. (2001). Perceptions of intentional wrongdoing and peer reporting behavior among registred nurses. Journal of Business Ethics, 34, 1-13.

Lopez, S., Calugaru, V., Lamproglou, I., Boskos, C., Taillibert, S., Simon, J. M., et al. (2008). Les effets de la liste d'attente sur la survie des patients atteints de glioblastome traité par irradiation. Cancer/ Radiothérapie, 12, 497-499.

MeaH. (2007). Améliorer la sécurité en radiothérapie. Paris: Mission nationale d'expertise et d'audit hospitaliers.

Miceli, M. P., Near, J. P., \& Dworkin, T. M. (2008). Whistle-blowing in Organisations. New York: Routledge.

Mollo, V., \& Falzon, P. (2004). Auto- and allo-confrontation as tools for reflective activities. Applied Ergonomics, 35(6), 531-540.

Nascimento, A., \& Falzon, P. (2008a). Risk management in Radiotherapy. In L. Sznelwar, F. Mascia, \& U. Montedo (Eds.), Proceedings of Human Factors in Organizational Design and Management - IX, 19-21 march. Sao Paulo, Brazil: Editora Blücher.

Nascimento, A., \& Falzon, P. (2008b). Concevoir la sécurité: la chaîne de traitement en radiothérapie. Paper presented at the XXXXIIIème Congrès de la SELF, Ajaccio, France.

Pavageau, P., Nascimento, A., \& Falzon, P. (2007). Les risques d'exclusion dans un contexte de transformation organisationnelle. PISTES, 9(2). http:/www.pistes.uqam.ca/v9n2/articles/v9n2a6.htm

Peiffert, D., Simon, J. M., \& Eschwege, F. (2007). L'accident d'Épinal : passé, présent, avenir. Cancer/ Radiothérapie, 11(6-7), 309-312.

Pidgeon, N., \& O’Leary, M. (1994). Organizational safety culture: implications for aviation pratice. In N. A. John, N. McDonald, \& R. Fuller (Eds.), Aviation psychology in pratice (pp. 21-43). Aldershot: Avebury Technical.

Pronovost, P., \& B. Sexton (2005). Assessing safety culture: guidelines and recommendations. Quality \& Safety in Health Care, 14, 231-233.

Rosenwald, J. C. (2002). Sécurité en radiothérapie: le contrôle des logiciels et des systèmes informatiquesSafety in radiation therapy: quality assurance of computerized system. Cancer/ Radiothérapie, 6(Supplement 1), 180-189.

SFRO. (2008). Guide des procédures de radiothérapie externe 2007. Cancer/Radiothérapie, 12(3-4), 143313.

Toupin, C. (2008). Expérience et redéfinition de la tâche dans le travail des infirmières de nuit: Une recherche menée dans des unités de pneumologie. Thèse d'Ergonomie, Cnam, Paris.

Woods, D. D. (2006). Essential characteristics of resilience. In E. Hollnagel, Woods D. D., \& Leveson N. (Eds.), Resilience engineering: Concepts and precepts (pp. 21 - 33). Aldershot, UK: Ashgate.

\section{RÉSUMÉ}

L'objectif du travail des manipulatrices en radiothérapie est double et consiste à assurer l'administration des soins journaliers, évitant les annulations de séances (produire la santé des patients) tout en s'assurant que l'administration des soins se fasse dans les conditions prescrites, évitant les incidents/accidents (produire la sécurité des patients). Un conflit entre ces deux objectifs apparaît en cas de situations non nominales. L'objectif de cet article est de mettre en évidence, à partir l'analyse de la gestion de 59 situations non nominales, les compromis dans le risque réalisés par les manipulatrices en radiothérapie afin de gérer ce conflit. Dans 47 des 59 situations non nominales, les manipulatrices ont géré le conflit de buts sans avoir à prendre des « décisions de sacrifice » visant 
à favoriser l'un des deux buts. Dans les 12 situations restantes, la gestion du conflit s'est faite par un compromis entre les objectifs de santé et de sécurité des patients. Lorsqu'elles prennent des décisions de sacrifice, les manipulatrices considèrent d'une part les risques et les bénéfices escomptés pour les patients, et d'autre part la phase de traitement, la connaissance des collègues et la satisfaction des patients.

\section{MoTS-CLÉ}

Conflit, compromis, récupération, risque, santé, sécurité, manipulatrices, patients, radiothérapie

\section{RÉFÉRENCEMENT}

Nascimento, A. \& Falzon, P. (2009). Produire la santé, produire la sécurité: récupérations et compromis dans le risque des manipulatrices en radiothérapie, Activités, 6 (2), pp. 3-23. http://www.activites.org/v6n2/ v6n2.pdf

Article soumis le 24 décembre 2008 accepté pour publication le 21 avril 2009 